PREPARED FOR THE U.S. DEPARTMENT OF ENERGY, UNDER CONTRACT DE-AC02-76CH03073

PPPL-3993

PPPL-3993

UC-70

Multispecies Density and Temperature Gradient

Dependence of Quasilinear Particle and Energy Fluxes

by

G. Rewoldt, R.V. Budny, and W.M. Tang

August 2004

$N_{\substack{\text { PRInCETOn PLASMA } \\ \text { PHYSIES LABORATORY }}}^{D}$

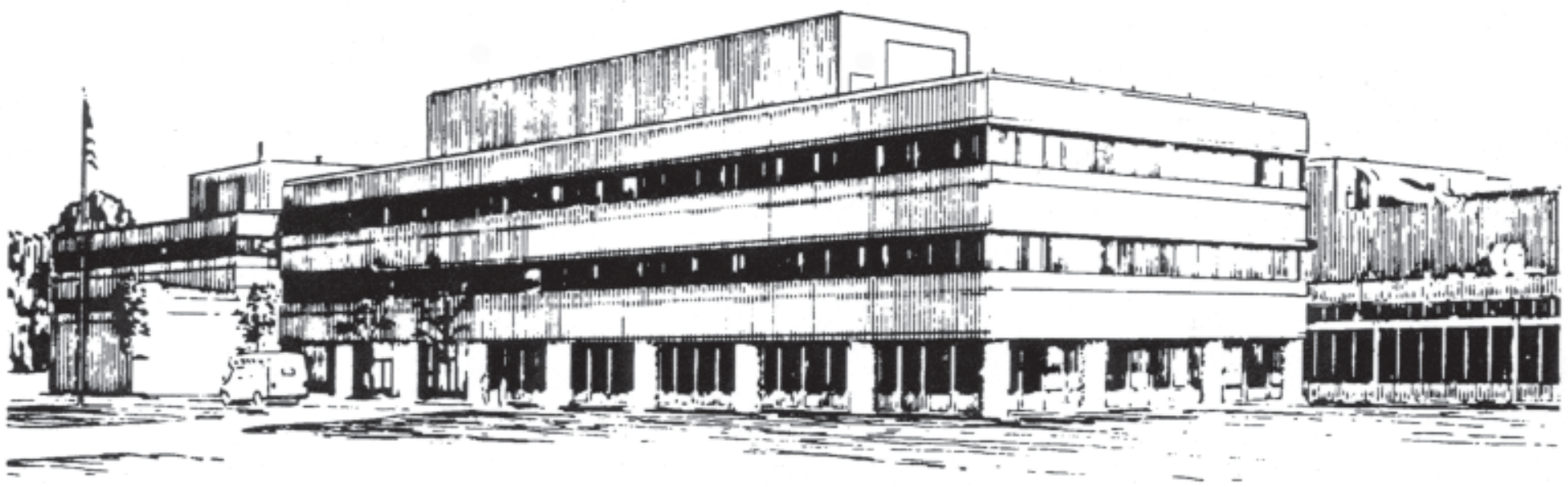

PRINCETON PLASMA PHYSICS LABORATORY PRINCETON UNIVERSITY, PRINCETON, NEW JERSEY 


\section{PPPL Reports Disclaimer}

This report was prepared as an account of work sponsored by an agency of the United States Government. Neither the United States Government nor any agency thereof, nor any of their employees, makes any warranty, express or implied, or assumes any legal liability or responsibility for the accuracy, completeness, or usefulness of any information, apparatus, product, or process disclosed, or represents that its use would not infringe privately owned rights. Reference herein to any specific commercial product, process, or service by trade name, trademark, manufacturer, or otherwise, does not necessarily constitute or imply its endorsement, recommendation, or favoring by the United States Government or any agency thereof. The views and opinions of authors expressed herein do not necessarily state or reflect those of the United States Government or any agency thereof.

\section{Availability}

This report is posted on the U.S. Department of Energy's Princeton Plasma Physics Laboratory Publications and Reports web site in Fiscal Year 2004. The home page for PPPL Reports and Publications is: http://www.pppl.gov/pub_report/

DOE and DOE Contractors can obtain copies of this report from:

U.S. Department of Energy

Office of Scientific and Technical Information

DOE Technical Information Services (DTIS)

P.O. Box 62

Oak Ridge, TN 37831

Telephone: (865) 576-8401

Fax: (865) 576-5728

Email: reports@adonis.osti.gov

This report is available to the general public from:

National Technical Information Service

U.S. Department of Commerce

5285 Port Royal Road

Springfield, VA 22161

Telephone: $1-800-553-6847$ or

(703) $605-6000$

Fax: (703) 321-8547

Internet: http://www.ntis.gov/ordering.htm 


\title{
Multispecies density and temperature gradient dependence of quasilinear particle and energy fluxes
}

\author{
G. Rewoldt,* R.V. Budny, and W.M. Tang \\ Princeton Plasma Physics Laboratory, \\ Princeton University, Princeton, New Jersey 08543-0451
}

\begin{abstract}
The variations of the normalised quasilinear particle and energy fluxes with artificial changes in the density and temperature gradients, as well as the variations of the linear growth rates and real frequencies, for ion temperature gradient and trapped-electron modes, are calculated. The quasilinear fluxes are normalised to the total energy flux, summed over all species. Here, realistic cases for tokamaks and spherical torii are considered which have two impurity species. For situations where there are substantial changes in the normalised fluxes, the "diffusive approximation," in which the normalised fluxes are taken to be linear in the gradients, is seen to be inaccurate. Even in the case of small artificial changes in density or temperature gradients, changes in the fluxes of different species ("off-diagonal") generally are significant, or even dominant, compared to those for the same species ("diagonal").
\end{abstract}

PACS numbers: 52.35.Qz,52.65.Tt,52.55.Fa 


\section{INTRODUCTION}

The FULL code ${ }^{1,2}$ can calculate the linear eigenfrequencies and eigenfunctions of high- $n$ instabilities in tokamaks and spherical torii and other devices, using the ballooning formalism so that the calculation is radially local. It can also calculate the corresponding quasilinear particle and energy fluxes for each species included in the calculation, using the converged eigenfrequency-eigenfunction results, and the fluxes are proportional to the square of the saturation level of the perturbed electrostatic potential for the instability. In the linear and quasilinear limit, this saturation level is unknown, but if ratios of quasilinear fluxes are taken, the unknown saturation level divides out. In the present work, the dependence of the quasilinear particle flux $\Gamma_{j}$ and total energy flux $Q_{j}$ (including any convective part) for species $j$ on the density gradient $d n_{j} / d r$ and the temperature gradient $d T_{j} / d r$, both of the

same species ("diagonal") and of different species ("off-diagonal"), will be examined for two experimentally-realistic cases.

The particle fluxes will be normalized to $Q_{t o t} / T_{j}$ and the energy fluxes to $Q_{t o t}$, where $Q_{t o t} \equiv \sum_{j} Q_{j}$, and the sum is over the electrons and all of the ion species. The resulting dimensionless normalized fluxes, independent of the unknown nonlinear saturation level, will be examined for their variation with the density and temperature gradients of each of the species from their experimental values.

Among other things, this variation will allow us to examine the accuracy of the "diffusive approximation," in which the flux is considered to be linear in the corresponding gradient. This diffusive approximation means, in the most simplified case with a single (density) gradient and a single (particle) flux, that $\Gamma(d n / d r)=n V-D(d n / d r)$, where $V$ and $D$ are considered to be independent of $(d n / d r)$. This expression can be thought of as a power series expansion of $\Gamma(d n / d r)$ in $d n / d r$, truncated after the first two terms. Here, we will examine the validity of the generalization of this approximation, to both density and temperature gradients and to multiple species, for the normalized quasilinear flux results, which are general nonlinear functions of all of the gradients.

The experimental cases considered are described in Sec. II, the different unstable modes and the roots of the eigenmode equation are discussed in Sec. III, and the constraints on the input gradients for the calculation and on the resultant fluxes are explained in Sec. IV. Then, the results of the separate artificial variation of each density and temperature gradient 
are described in Sec. V. Limited comparisons of the calculated normalized fluxes with the corresponding experimentally-derived normalized fluxes are made in Sec. VI, and general trends and conclusions are given in Sec. VII.

\section{CASES}

We will consider a case for the Joint European Torus ${ }^{3}$ (JET) tokamak and a case for the National Spherical Torus Experiment ${ }^{4}$ (NSTX) spherical torus. JET and NSTX discharges of the types considered here are discussed from an experimental point of view in Refs. 5 and 6 , respectively. The JET case corresponds to JET discharge 53030 at $t=21.5 \mathrm{~s}$, and the NSTX case corresponds to discharge 108213 at $t=0.320 \mathrm{~s}$, but using the neon density profile measured for the nominally identical discharge 108216 at that time. JET discharge 53030 is an ELMy H-mode plasma with argon seeding, with global parameters $R=2.9 \mathrm{~m}, a=0.9$ $\mathrm{m}, I_{p}=2.5 \mathrm{MA}, B_{Z}=2.5 \mathrm{~T}, P_{N B}=12.3 \mathrm{MW}, T_{i}(0)=T_{C}(0)=2.6 \mathrm{keV}, T_{e}(0)=2.9 \mathrm{keV}$, and $n_{e}(0)=1.1 \times 10^{20} \mathrm{~m}^{-3}$. NSTX discharge 108213 is an L-mode plasma without neon injection and NSTX discharge 108216 is an L-mode plasma with neon injection, both with global parameters $R=0.87 \mathrm{~m}, a=0.65 \mathrm{~m}, I_{p}=1.0 \mathrm{MA}, B_{Z}=0.44 \mathrm{~T}, P_{N B}=1.6 \mathrm{MW}$, $T_{i}(0)=T_{C}(0)=1.6 \mathrm{keV}, T_{e}(0)=1.2 \mathrm{keV}$, and $n_{e}(0)=4.1 \times 10^{19} \mathrm{~m}^{-3}$. In addition to the usual electron (e), background thermal deuterium ion $(i)$, carbon impurity $\left(C, Z_{C}=6\right)$, and hot deuterium beam $(b)$ species, each of these cases includes a second heavier impurity species with a very small density fraction, argon $\left(A r, Z_{A r}=18\right)$ for the JET case and neon $\left(N e, Z_{N e}=10\right)$ for the NSTX case. We approximate the impurity species for each case as fully stripped, though this will not quite be achieved in the experiments at the radii of interest. All of the species are employed with Maxwellian equilibrium distribution functions. We choose $r / a=0.750$ and $k_{\theta} \rho_{i}=0.50$ for the "hybrid" root for the JET case, and we choose $r / a=0.720$ and $k_{\theta} \rho_{i}=0.262$ for the ion temperature gradient (ITG) mode root for the NSTX case, and $r / a=0.730$ and $k_{\theta} \rho_{i}=0.248$ for the trapped-electron mode (TEM) root for the NSTX case, since these values approximately maximize the linear growth rates of the instabilities considered. These different roots and their relationships will be discussed in Sec. III.

The experimentally-reconstructed magnetohydrodynamic (MHD) equilibria for these cases are calculated numerically in flux coordinates, using the experimentally measured 
or calculated density and temperature profiles for each species. The MHD equilibria include finite- $\beta$ effects such as the Shafranov shift. The flux surface label $r / a$ is defined in terms of the normalized toroidal flux, $r / a \equiv \sqrt{\Phi / \Phi_{a}}$, where $a$ refers to the plasma boundary. Thus, $r$ is only approximately a geometrical length, but specifically refers to this function of the toroidal flux. When the density and temperature gradients of each species are artificially varied from their experimental values in the FULL code instability calculation, the MHD equilibrium is held fixed; in this sense the calculation is not self-consistent. Also, in experimental plasmas, the local value and the local gradient of a plasma parameter are highly correlated, but here we are changing only the individual gradients, in the previous artificial sense of Green and Chance. ${ }^{7}$

The local experimental parameters for the JET case at $r / a=0.75$ are: $n_{e}=6.30 \times 10^{19}$ $\mathrm{m}^{-3}, T_{e}=1.40 \mathrm{keV}, T_{i}=1.27 \mathrm{keV}, T_{C}=T_{A r}=1.27 \mathrm{keV}, T_{b}=23.1 \mathrm{keV}, n_{i} / n_{e}=0.907$, $n_{C} / n_{e}=0.0141, n_{A r} / n_{e}=0.0000614, n_{b} / n_{e}=0.00764, r_{n i} / r_{n e}=1.12, r_{n C} / r_{n e}=0.473$, $r_{n A r} / r_{n e}=9.89, r_{n b} / r_{n e}=0.575, \eta_{e}=1.99, \eta_{i}=3.04, \eta_{C}=1.28, \eta_{A r}=26.7, \eta_{b}=0.486$, and $q=2.01$. Here, $r_{n j} \equiv-\left(d \ln n_{j} / d r\right)^{-1}$ and $\eta_{j} \equiv\left(d \ln T_{j} / d r\right) /\left(d \ln n_{j} / d r\right)$, and the other notation is standard.

The local experimental parameters for the ITG root for the NSTX case at $r / a=0.72$ are: $n_{e}=1.87 \times 10^{19} \mathrm{~m}^{-3}, T_{e}=0.179 \mathrm{keV}, T_{i}=0.180 \mathrm{keV}, T_{C}=T_{N e}=0.180 \mathrm{keV}$, $T_{b}=18.5 \mathrm{keV}, n_{i} / n_{e}=0.947, n_{C} / n_{e}=0.00534, n_{N e} / n_{e}=0.00187, n_{b} / n_{e}=0.00192$, $r_{n i} / r_{n e}=1.10, r_{n C} / r_{n e}=0.403, r_{n N e} / r_{n e}=0.364, r_{n b} / r_{n e}=0.192, \eta_{e}=1.91, \eta_{i}=2.29$, $\eta_{C}=0.835, \eta_{N e}=0.755, \eta_{b}=-0.169$, and $q=2.12$. The local experimental parameters for the TEM root for the NSTX case at $r / a=0.73$ are: $n_{e}=1.82 \times 10^{19} \mathrm{~m}^{-3}, T_{e}=0.170 \mathrm{keV}$, $T_{i}=0.170 \mathrm{keV}, T_{C}=T_{N e}=0.170 \mathrm{keV}, T_{b}=18.9 \mathrm{keV}, n_{i} / n_{e}=0.950, n_{C} / n_{e}=0.00516$, $n_{N e} / n_{e}=0.00177, n_{b} / n_{e}=0.00170, r_{n i} / r_{n e}=1.09, r_{n C} / r_{n e}=0.503, r_{n N e} / r_{n e}=0.321$, $r_{n b} / r_{n e}=0.192, \eta_{e}=1.92, \eta_{i}=2.19, \eta_{C}=1.01, \eta_{N e}=0.645, \eta_{b}=-0.178$, and $q=2.20$.

\section{MODES AND ROOTS}

The linear instability calculation is performed with the FULL code in the electrostatic limit, without rotation, including collisions, for $k_{\theta} \rho_{i} \lesssim 1$, but with $n \gg 1$. In this limit, there are two modes of interest: the ion temperature gradient (ITG) mode and the trappedelectron mode (TEM), which normally have real frequencies $\omega_{r}$ in the ion and the electron 
diamagnetic directions, respectively. Depending on the parameters, these two modes can remain as separate roots of the eigenmode equation, as happens here for the NSTX case, or can "hybridize" into a single root, as happens here for the JET case. For a hybrid root, the real frequency $\omega_{r}$ will normally make a transition from the electron diamagnetic direction to the ion diamagnetic direction as $k_{\theta} \rho_{i}$ increases, and the hybrid root correspondingly goes from a TEM-like regime to an ITG-like regime.

The behaviour of the roots depends strongly on the parameter $\eta_{i}^{e} \equiv$ $\left(d \ln T_{i} / d r\right) /\left(d \ln n_{e} / d r\right)$. Increasing $\eta_{i}^{e}$ will also normally move the real frequency from the electron diamagnetic direction to the ion diamagnetic direction; $\eta_{i}^{e}$ can be changed either by varying $\left(d T_{i} / d r\right)$ or by varying $\left(d n_{e} / d r\right)$, but it will be seen that the two kinds of variation are not equivalent.

\section{CONSTRAINTS}

There are constraints both on the input to and on the output of the calculation. The input constraints come from the requirement of electric charge neutrality on every magnetic surface. Not only does this require that $\sum_{j} Z_{j} n_{j}=0$ on the chosen magnetic surface for the calculation, but the radial derivative of this charge neutrality condition also imposes a condition on the density gradients of the different species. If the density gradients of all but one of the species are chosen freely, then this condition determines the value of the density gradient of the remaining species. As a matter of convention, this adjusted species is taken to be the background thermal deuterium ion species, though this is an arbitrary choice (and the results would be somewhat different with a different choice). Thus, as other species are turned on or off in the FULL code, or as the densities or density gradients of other species are changed, the density and density gradient of the background thermal deuterium ion species are adjusted to satisfy the requirements of charge neutrality and of the radial derivative of charge neutrality on the chosen magnetic surface.

There is also a constraint on the output of the calculation, in particular on the quasilinear particle fluxes. It was shown in Ref. 2 that these modes satisfying the quasineutrality condition cause no net flux of electric charge, that is $\sum_{j} Z_{j} \Gamma_{j}=0$, and the transport is thus automatically ambipolar. When the eigenfrequency and eigenfunction satisfy the eigenmode equation to good accuracy, this ambipolarity condition is also satisfied to good 
accuracy. Thus, when some parameter is changed, and the particle fluxes of the other species change correspondingly, the particle flux of one species (again, say the background thermal deuterium ion species) can be regarded as adjusting to satisfy this ambipolarity condition.

\section{RESULTS}

In this section we examine the effects of artificial gradient variation on the linear growth rates and real frequencies of the different modes, and on the corresponding normalized quasilinear particle and energy fluxes for the five species. There are four independent density gradients, as explained in Sec. IV, and five independent temperature gradients, which will generally be varied from zero up to twice the corresponding experimental values, so that there are nine gradients to be varied in all, and the results are shown in nine figures. There are five particle fluxes (four independent, as explained in Sec. IV), and five energy fluxes for each root, for each set of input gradients. We will vary the input gradients roughly in order from those which have the largest effect on the normalized fluxes, to those with successively smaller effects. For the JET case, there is only one "hybrid" root, and thus only one $Q_{t o t}$, but for the NSTX case there are separate ITG and TEM roots, so we use separate $Q_{t o t}^{I T G}$ and $Q_{t o t}^{T E M}$ normalizations for them, and these can in general be different.

The subfigures in the left-hand column for each figure are for the JET case and those in the right-hand column are for the NSTX case. The top pair of subfigures in each figure shows the growth rate $\gamma$ and the real frequency $\omega_{r}$, the middle pair of subfigures shows the normalized particle fluxes $\Gamma_{j} T_{j} / Q_{t o t}$, and the bottom pair of subfigures shows the normalized total energy fluxes $Q_{j} / Q_{t o t}$. The growth rates are shown as solid lines and the real frequencies as dashed lines, the JET hybrid root fluxes and the NSTX ITG mode fluxes as solid lines and the NSTX TEM mode fluxes as dashed lines. The color coding is that the JET hybrid mode and the NSTX ITG mode eigenfrequencies are in black, the NSTX TEM mode eigenfrequencies are in yellow, the electron $(e)$ fluxes are in magenta, the background thermal deuterium ion $(i)$ fluxes are in red, the carbon $(C)$ fluxes are in green, the argon $(A r)$ or neon $(N e)$ fluxes are in cyan, and the hot deuterium beam (b) fluxes are in blue. 


\section{A. Ion temperature gradient}

In Fig. 1 the results of varying the background thermal deuterion ion (ion) species temperature gradients from zero to two times the experimental value for the JET case, and from zero to three times the experimental value for the NSTX case, are shown. In Fig. 1(a) for the JET case, only the ITG-like range of the JET hybrid root is seen; the TEM-like range will be seen when other gradients are varied. In Fig. 1(b) for the NSTX case, the NSTX TEM root has very small growth rate for the experimental parameters, but acquires a larger growth rate as the ion temperature gradient is decreased or, as will be seen, the electron density gradient is increased. Also, the NSTX ITG root growth rate increases as the ion temperature gradient increases above a threshold value.

In Fig. 1(c) and (d), the normalized electron particle fluxes decrease with increasing ion temperature gradient, gradually for the JET case, and gradually, then steeply after a root transition, and then gradually again for the NSTX case. The carbon particle flux decreases, for increasing ion temperature gradient above a certain value, while the argon, neon, and beam species particle fluxes are always small. The ion particle flux is positive (outward), then negative (inward), and then positive again, for both cases, with increasing ion temperature gradient, to balance the particle fluxes of the other species so as to give zero net electric charge flux, as explained in Sec. IV. Note that any change in the carbon particle flux has to be balanced by a six-times larger change in the ion particle flux to satisfy this condition, and correspondingly for the argon and neon species.

Note also that the scales on the particle flux Figs. 1(c) and (d) are different from the scales for the energy flux Figs. 1(e) and (f). The particle fluxes $\Gamma_{j}$ are always small compared to $Q_{t o t} / T_{j}$ for all species, while the largest $Q_{j}$ for any species can be comparable to $Q_{t o t}$. This will be seen to be true regardless of which gradient is being varied.

For both cases, as the ion temperature gradient is increased above a certain value, the normalized ion energy flux increases, while the normalized electron and carbon energy fluxes decrease, though more steeply for the NSTX case than for the JET case, and the normalized argon or neon and beam energy fluxes are always small. The beam particle and energy fluxes are small not only because of the small beam density fraction, but also because the beam particles interact weakly with these modes. The bounce frequencies for trapped beam particles and the transit frequencies for untrapped beam particles are large compared to the 
mode frequencies for all of the roots, and this causes weak interaction of these modes with the beam species.

These results for $\Gamma_{j} T_{j} / Q_{t o t}$ and $Q_{j} / Q_{t o t}$ as functions of $d T_{i} / d r$ all seem to have a substantial amount of "curvature," or departure from linearity, except when the amount of variation is small, and appear to be asymptoting to a constant value as $d T_{i} / d r$ reaches its maximum value in these plots. The corresponding variation in the unnormalized fluxes $\Gamma_{j}$ and $Q_{j}$ will be modified by whatever variation comes through the $Q_{t o t}$ factor for the respective root, and that is unknown in the present calculation.

\section{B. Electron density gradient}

The variation with the electron density gradient is shown in Fig. 2, from zero to three times the experimental value for the JET case, and from zero to two times the experimental value for the NSTX case. As $d n_{e} / d r$ increases from somewhat more than the experimental value in Fig. 2(a), for the JET case, the TEM-like range of the hybrid root occurs, with the real frequency making a transition into the electon diamagnetic direction. For the NSTX case in Fig. 2(b), the TEM root has a very small growth rate for the experimental value of $d n_{e} / d r$, but becomes strongly unstable as the density gradient increases. The ITG root is stabilized if the electron density gradient becomes either too large or too small compared to the experimental value.

As $d n_{e} / d r$ increases for the JET case in Fig. 2(c), the normalized electron particle flux increases gradually, then steeply, and then saturates. The carbon particle flux increases to a maximum, and then decreases, over the same range. To maintain ambipolarity, the ion particle flux is first positive, then negative, and then positive again. The argon and beam species fluxes are small. All of these statements hold also for the NSTX case in Fig. 2(d), except that there is a transition from the ITG root to the TEM root in the progression.

For the normalized energy fluxes in Fig. 2(e) for the JET case, $Q_{i} / Q_{t o t}$ decreases and $Q_{e} / Q_{t o t}$ increases as $d n_{e} / d r$ increases, with near saturation at the maximum of $d n_{e} / d r$; $Q_{C} / Q_{t o t}$ has a maximum near where $Q_{i} / Q_{t o t}$ and $Q_{e} / Q_{t o t}$ are changing most rapidly, which is where $\omega_{r}$ has the smallest magnitude (this allows stronger interaction of the mode with the carbon species). The beam and argon fluxes are small. Again, these statements apply also for the NSTX case in Fig. 2(f), but again with the ITG to TEM root transition. 
Also, $\Gamma_{N e}^{I T G} T_{N e} / Q_{t o t}^{I T G}$ and $\Gamma_{C}^{I T G} T_{C} / Q_{t o t}^{I T G}$, as well as $Q_{N e} / Q_{t o t}$ and $Q_{C} / Q_{t o t}$, change substantially just before the ITG root goes stable with increasing $d n_{e} / d r$, but $Q_{t o t}$ would presumably be getting small close to marginal stability also, so there would probably be no corresponding substantial increases in $\Gamma_{N e}, \Gamma_{C}, Q_{N e}$, and $Q_{C}$ themselves.

\section{Electron temperature gradient}

The effects of varying electron temperature gradient are somewhat weaker than those of varying ion temperature gradient or of varying electron density gradient for these cases, as shown in Fig. 3. The JET case hybrid root is somewhat destabilized by increasing $d T_{e} / d r$, as is the NSTX case ITG root, while for the NSTX case the weak TEM root is stabilized by $d T_{e} / d r$ changing too much in either direction. The changes in the normalized particle and energy fluxes for the JET case are moderate, and the temperature gradient dependence is close to being linear. However, for the corresponding NSTX case fluxes, there is still noticable "curvature." With increasing $d T_{e} / d r$, there is an increase in $Q_{e} / Q_{t o t}$ and a corresponding decrease in $Q_{i} / Q_{t o t}$ for all roots, with moderate changes for the other species.

\section{Carbon density gradient}

Increasing carbon density gradient is stabilizing for all cases and roots here, as shown in Fig. 4. The normalized carbon particle and energy fluxes increase, with a corresponding decrease in the ion particle flux, satisfying the ambipolarity condition. The effects on the fluxes of other species is moderate or small.

\section{E. Carbon temperature gradient}

Increasing carbon temperature gradient has little effect on the growth rates, as shown in Fig. 5. As with the carbon density gradient, increasing carbon temperature gradient increases the normalized carbon particle and energy fluxes, with a corresponding decrease in the ion particle flux, with little change in the fluxes for the other species. 


\section{F. Argon and neon density gradient}

The effects of varying the argon density gradient for the JET case and the neon density gradient for the NSTX case are shown in Fig. 6. The density fractions $n_{A r} / n_{e}$ and $n_{N e} / n_{e}$ are small, and the effects of the density gradient variation are correspondingly weak. Increasing $d n_{N e} / d r$ stabilizes the already very weak TEM root for the NSTX case. The changes in the normalized fluxes $\Gamma_{j} T_{j} / Q_{t o t}$ and $Q_{j} / Q_{t o t}$ are also very weak, except for the TEM root for the NSTX case just before the marginal stability point, and, in this range for $d n_{N e} / d r, Q_{t o t}$

should be going to zero, so that the corresponding unnormalized fluxes $\Gamma_{j}$ and $Q_{j}$ should also be going to zero.

\section{G. Argon and neon temperature gradient}

Again, the small density fractions $n_{A r} / n_{e}$ and $n_{N e} / n_{e}$ give small or moderate changes in the eigenfrequencies and normalized fluxes when the argon and neon temperature gradients are changed, as shown in Fig. 7.

\section{H. Beam density gradient}

The hot deuterium beam species density fraction $n_{b} / n_{e}$ is also small, and the changes in the growth rates and real frequencies and the absolute changes in the normalized particle and energy fluxes are small for all species, as shown in Fig. 8. However, the relative changes in the normalized beam species fluxes $\Gamma_{b} T_{b} / Q_{t o t}$ and $Q_{b} / Q_{\text {tot }}$ are large, of order unity, for this order unity change in $d n_{b} / d r$. This relative change is difficult to see in Fig. 8, because the absolute magnitudes of $\Gamma_{b} T_{b} / Q_{\text {tot }}$ and $Q_{b} / Q_{\text {tot }}$ are so small.

\section{Beam temperature gradient}

Finally, the effects of varying the beam species temperature gradients $d T_{b} / d r$ are shown in Fig. 9. Again, because of the small beam density fraction and the weak interaction of the beam particles with these roots, the changes in the eigenfrequencies and in the normalized particle and energy fluxes are small for all species, in an absolute sense, but are again 
substantial in a relative sense for $\Gamma_{b} T_{b} / Q_{t o t}$ and $Q_{b} / Q_{t o t}$ (though not as large as when varying $\left.d n_{b} / d r\right)$.

\section{EXPERIMENTAL COMPARISONS}

The TRANSP transport analysis $\operatorname{code}^{8}$ has been used to derive experimental particle and energy fluxes, from the time evolution of the experimental density and temperature profiles and the particle and energy sources and sinks, for the different plasma species, with some limitations for these cases. The experimentally-derived normalized particle fluxes $\Gamma_{j} T_{j} / Q_{t o t}$ are all small, as are also the corresponding calculated quasilinear normalized particle fluxes. However, the experimentally-derived fluxes are difficult to distinguish from zero, within the experimental uncertainties, so only the normalized energy fluxes will be compared quantitatively here. TRANSP can calculate only $Q_{e}$ and $Q_{\text {therm-ion, }}$, where $Q_{\text {therm-ion }} \equiv$ $Q_{i}+Q_{A r}+Q_{C}$ for the JET case and $Q_{t h e r m-i o n} \equiv Q_{i}+Q_{N e}+Q_{C}$ for the NSTX case. The experimentally-derived normalized energy fluxes are $Q_{e} / Q_{t o t} \simeq\left(1-Q_{\text {therm-ion }} / Q_{\text {tot }}\right)=0.1$ for the JET case and 0.85 for the NSTX case $\left(Q_{b} / Q_{t o t}\right.$ is negligable compared to $Q_{j} / Q_{t o t}$ for the other species, for both cases). The corresponding quasilinear normalized energy fluxes are $Q_{e} / Q_{t o t} \simeq\left(1-Q_{\text {therm-ion }} / Q_{t o t}\right)=0.2$ for the JET case hybrid root and 0.44 for the NSTX case for the dominant ITG root, for the experimental parameters. Thus, the quasilinear ratios differ from the corresponding experimentally-derived ratios by about a factor of two for both cases (but in opposite directions for the two cases). A factor of two disageement is not surprising, considering the approximations in the calculation here. The FULL code calculation is linear and quasilinear (a weak turbulence limit) and is radially local, while the experiments are in some sort of fully-turbulent state and include radially-nonlocal effects as well. In addition, each FULL code calculation only includes a single $k_{\theta} \rho_{i}$ value, while the experiments include contributions from a broad spectrum of fluctuations, including possibly much shorter and much longer wavelengths. Note also that the values of $k_{\theta} \rho_{i}$ that maximize the linear growth rates can shift somewhat away from the fixed values used here, as the individual density and temperature gradients are artificially varied. 


\section{GENERAL TRENDS AND CONCLUSIONS}

From the results presented in Sec. V, we can draw a number of general conclusions. (i) The range of density and temperature gradient variation surveyed here for the five species often involves regime change, between stable and unstable, or between ITG-like and TEM-like. (ii) Usually, when there are large changes in the gradients, the normalized fluxes $\Gamma_{j} T_{j} / Q_{t o t}$ and $Q_{j} / Q_{t o t}$ are not linear in the gradients, i.e., the diffusive approximation is not accurate in this situation. Of course, there would be additional gradient dependence in the unnormalized fluxes $\Gamma_{j}$ and $Q_{j}$ due to the gradient dependence of $Q_{t o t}$. (iii) The particle fluxes $\Gamma_{j}$ are always small compared to $Q_{t o t} / T_{j}$, and thus to the largest of the $Q_{j} / T_{j}$. (iv) For all the roots, the strongest overall dependence of the normalized fluxes is on the electron density gradient and the (background thermal deuterium) ion temperature gradient, even for the impurity and beam species. (v) The hot deuterium beam species normalized particle and energy fluxes are also strongly dependent on the beam species density gradient, and to a lesser extent, on the beam species temperature gradient. (vi) The background thermal deuterium ion particle flux is often inwards, to satisfy the condition of ambipolarity discussed in Sec. IV. (vii) The other fluxes are usually outward, except occasionally for the small argon or neon fluxes, and occasionally for the small beam fluxes. (viii) Despite the substantial differences between the JET and NSTX experiments, there appear to be many common qualitative trends in the effects of varying individual gradients for these cases, as described in Sec. V.

The breakdown of the "diffusive" approximation was previously investigated in another way in Ref. 9 by quantitatively evaluating the power-series coefficient matrices through second order (quadratic in the gradients). This was done in that reference for a simplified case with only three gradients and three fluxes, corresponding to a plasma with only electrons and background hydrogenic ions. It was concluded there that the quadratic contributions to the total fluxes were larger than the linear contributions, i.e., the power series for the case there appeared to be diverging, and not converging. That result is consistent with the present result that the departure of the multispecies fluxes from "linearity" in the gradients is large when the relative change in the normalized fluxes is substantial.

The experimentally-derived normalized electron energy fluxes for the two cases differ by about a factor of two from the corresponding quasilinear normalized electron energy fluxes. This is not surprising, considering the limitations of the calculation employed here. The 
results presented here are in the linear and quasilinear limit, and are only for a single flux surface and a single value of $k_{\theta} \rho_{i}$ for each root, and the behaviour over a wider range in $r / a$ or in $k_{\theta} \rho_{i}$ could be different. In the future, radially-global nonlinear simulations should be able to provide much better calculated information about the density and temperature profile dependence of the flux profiles for each species.

\section{Acknowledgments}

The authors would like to thank Dr. D. Stutman for providing the input data for the NSTX case. This work was supported by U.S. DOE Contract No. DE-AC02-76-CHO-3073.

* rewoldt@pppl.gov

1 G. Rewoldt, W. M. Tang, and M. S. Chance, Phys. Fluids 25, 480 (1982).

2 G. Rewoldt, W. M. Tang, and R. J. Hastie, Phys. Fluids 30, 807 (1987).

3 P. H. Rebut and B. E. Keen, Fusion Technol. 11, 13 (1987).

4 M. Ono, S. M. Kaye, Y.-K. M. Peng, et al, Nucl. Fusion 40, 557 (2000).

5 J. Ongena, P. Monier-Garbet, W. Suttrop, et al, Nucl. Fusion 44, 124 (2004); P. Dumortier, P. Andrew, G. Bonheure, et al, Plasma Phys. Control. Fusion 44, 1845 (2002).

6 D. Stutman, M. Finkenthal, R. E. Bell, et al, Phys. Plasmas 10, 4387 (2003).

7 J. M. Greene and M. S. Chance, Nucl. Fusion 21, 453 (1981).

8 R. Goldston, Basic Physical Processes of Toroidal Fusion Plasmas (Proc. Course and Workshop, Varenna, 1985), Vol. 1, p. 165 (Monotypia Franchi, Città di Castello, 1985).

9 See National Technical Information Service Document No. PPPL-2650, by G. Rewoldt (Princeton Plasma Physics Laboratory Report PPPL-2650, 1989). 


\section{FIGURE CAPTIONS}

FIG. 1: Variation of the growth rate, real frequency, normalized particle fluxes, and normalized energy fluxes with variation of the background thermal deuterium ion temperature gradient.

FIG. 2: Variation of the growth rate, real frequency, normalized particle fluxes, and normalized energy fluxes with variation of the electron density gradient.

FIG. 3: Variation of the growth rate, real frequency, normalized particle fluxes, and normalized energy fluxes with variation of the electron temperature gradient.

FIG. 4: Variation of the growth rate, real frequency, normalized particle fluxes, and normalized energy fluxes with variation of the carbon density gradient.

FIG. 5: Variation of the growth rate, real frequency, normalized particle fluxes, and normalized energy fluxes with variation of the carbon temperature gradient.

FIG. 6: Variation of the growth rate, real frequency, normalized particle fluxes, and normalized energy fluxes with variation of the argon or neon density gradient.

FIG. 7: Variation of the growth rate, real frequency, normalized particle fluxes, and normalized energy fluxes with variation of the argon or neon temperature gradient.

FIG. 8: Variation of the growth rate, real frequency, normalized particle fluxes, and normalized energy fluxes with variation of the hot deuterium beam density gradient.

FIG. 9: Variation of the growth rate, real frequency, normalized particle fluxes, and normalized energy fluxes with variation of the hot deuterium beam temperature gradient. 
JET
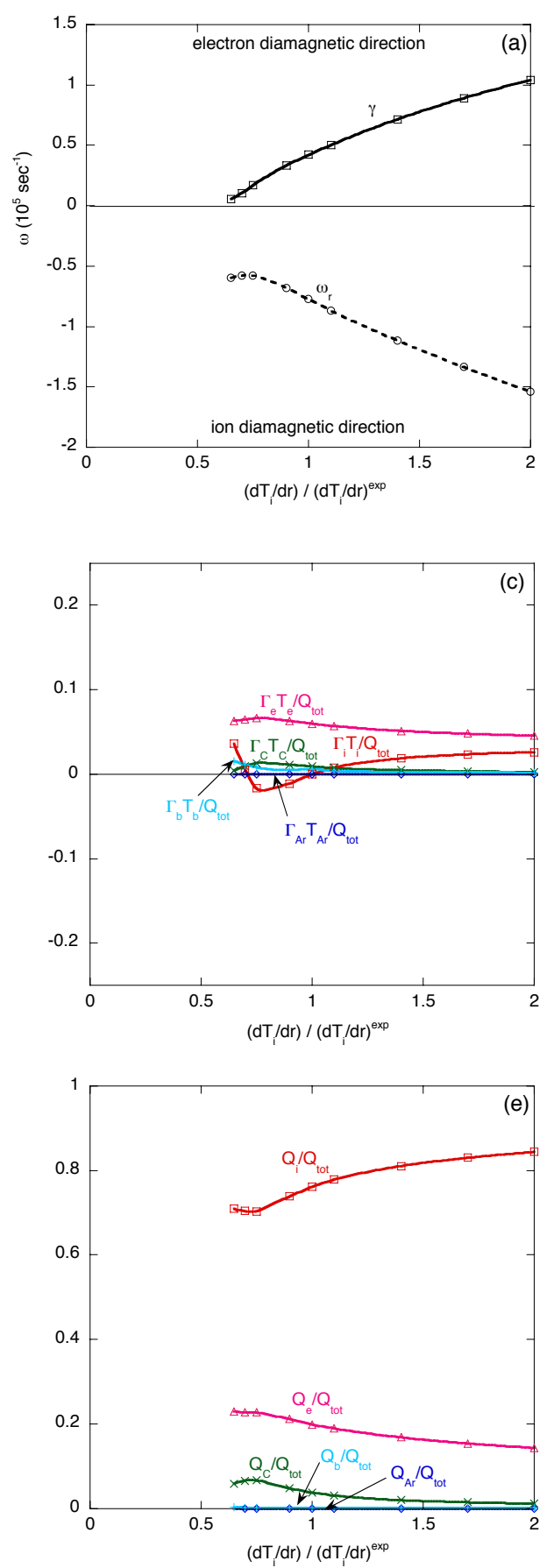

NSTX
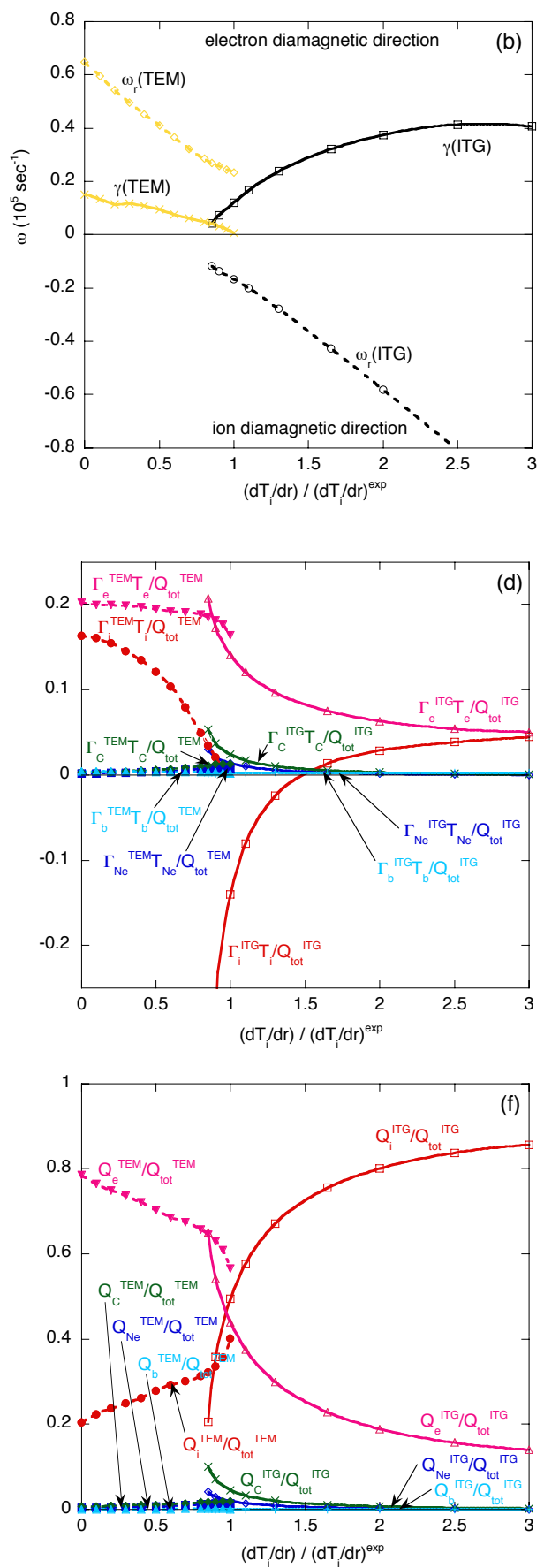

Fig. 1 

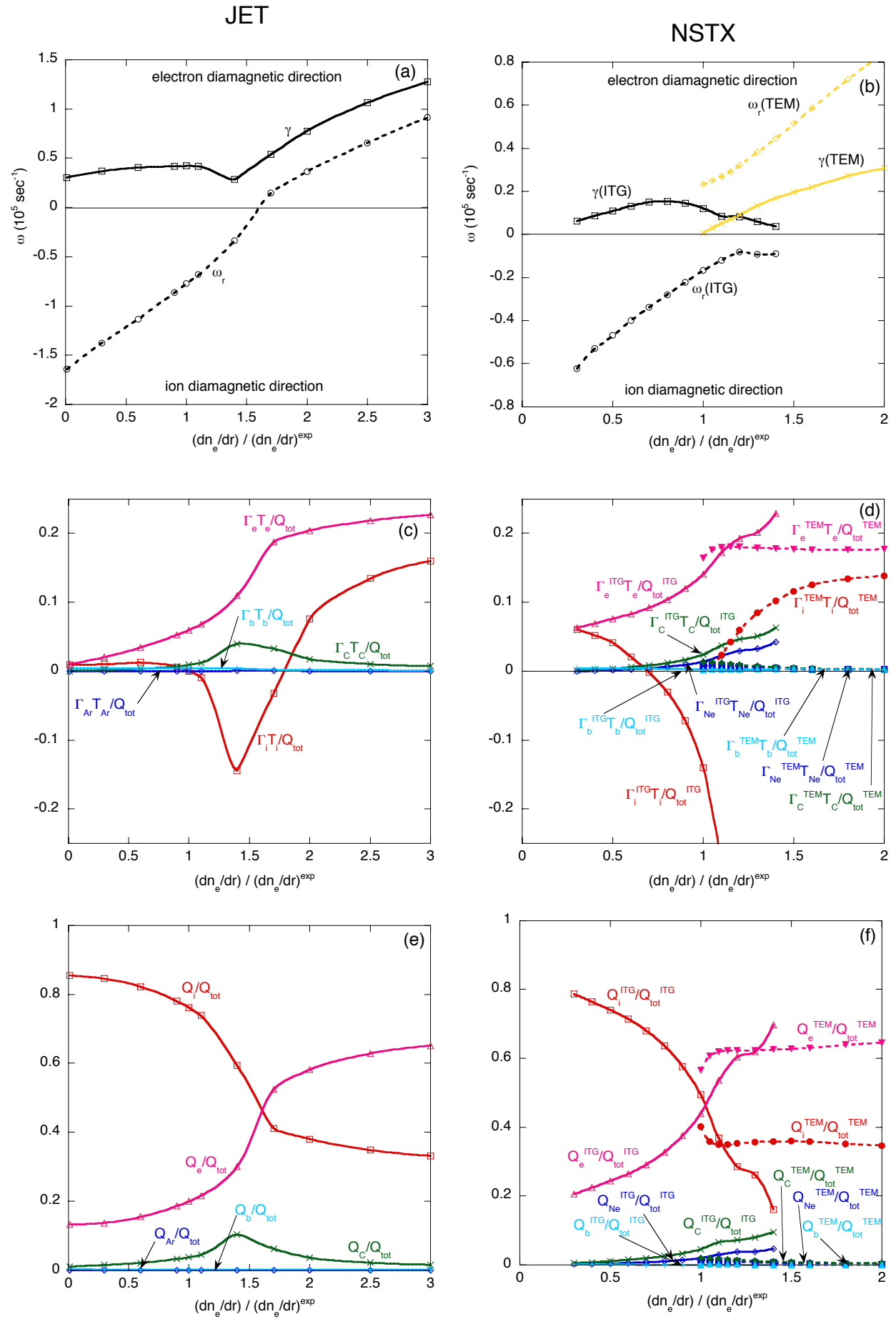

Fig. 2 
JET
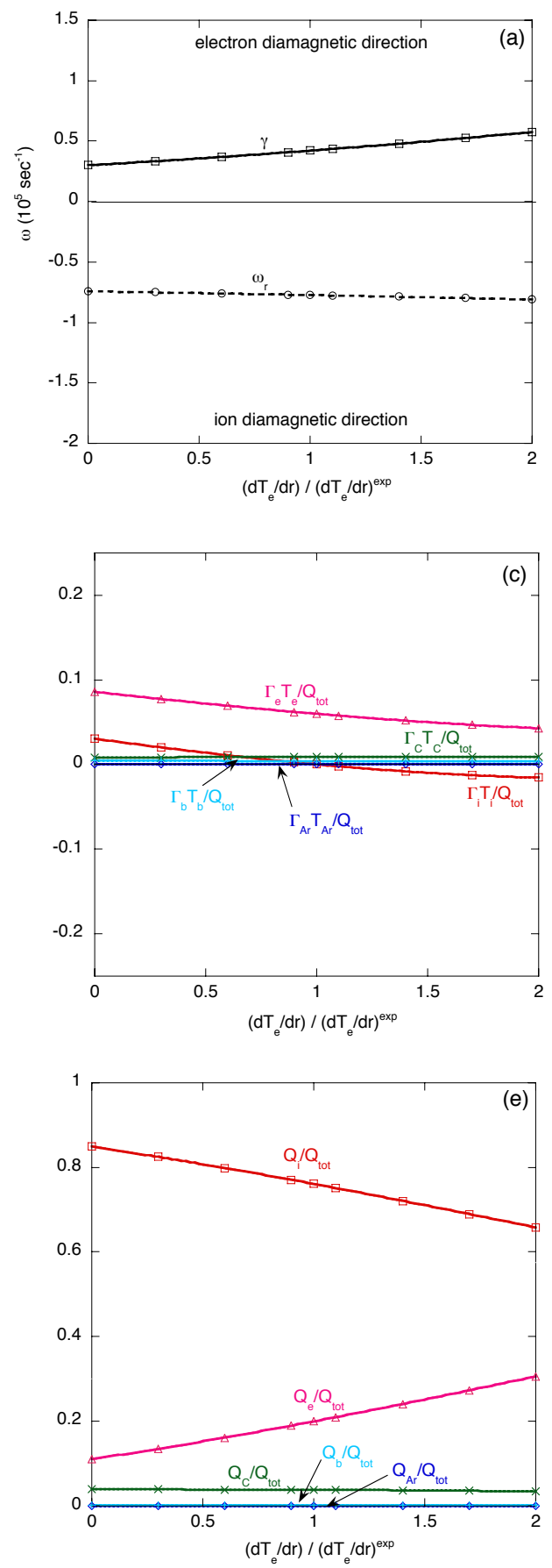

NSTX
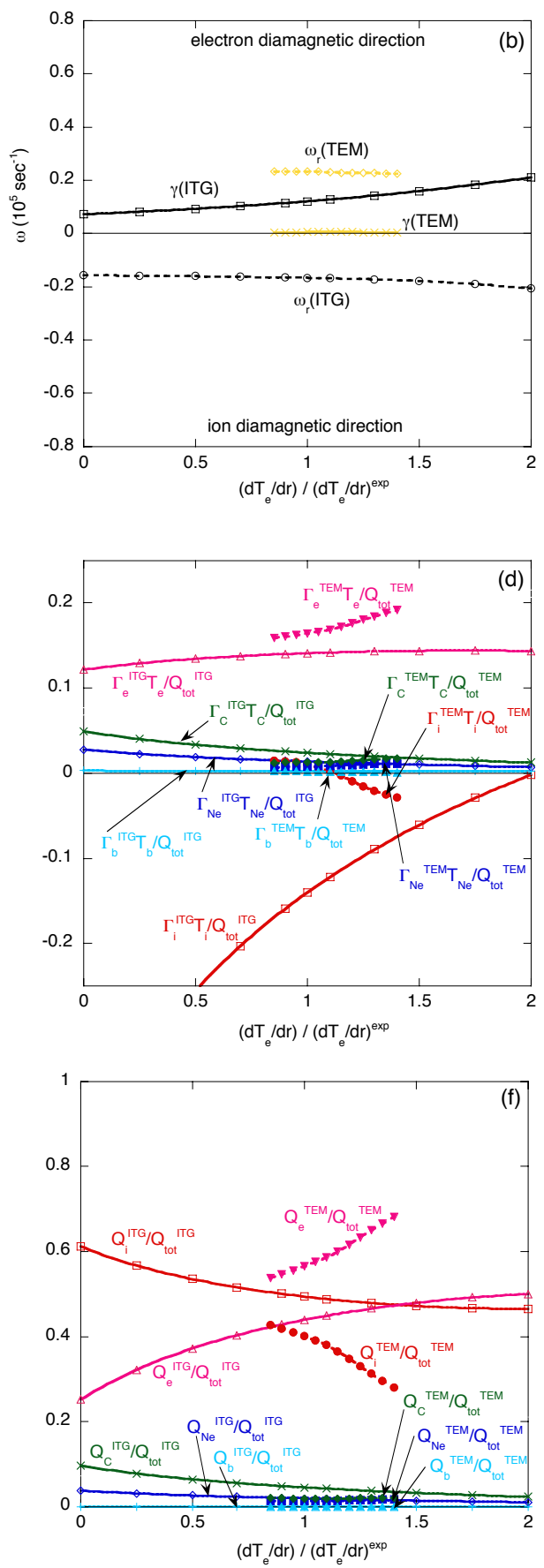

Fig. 3 
JET
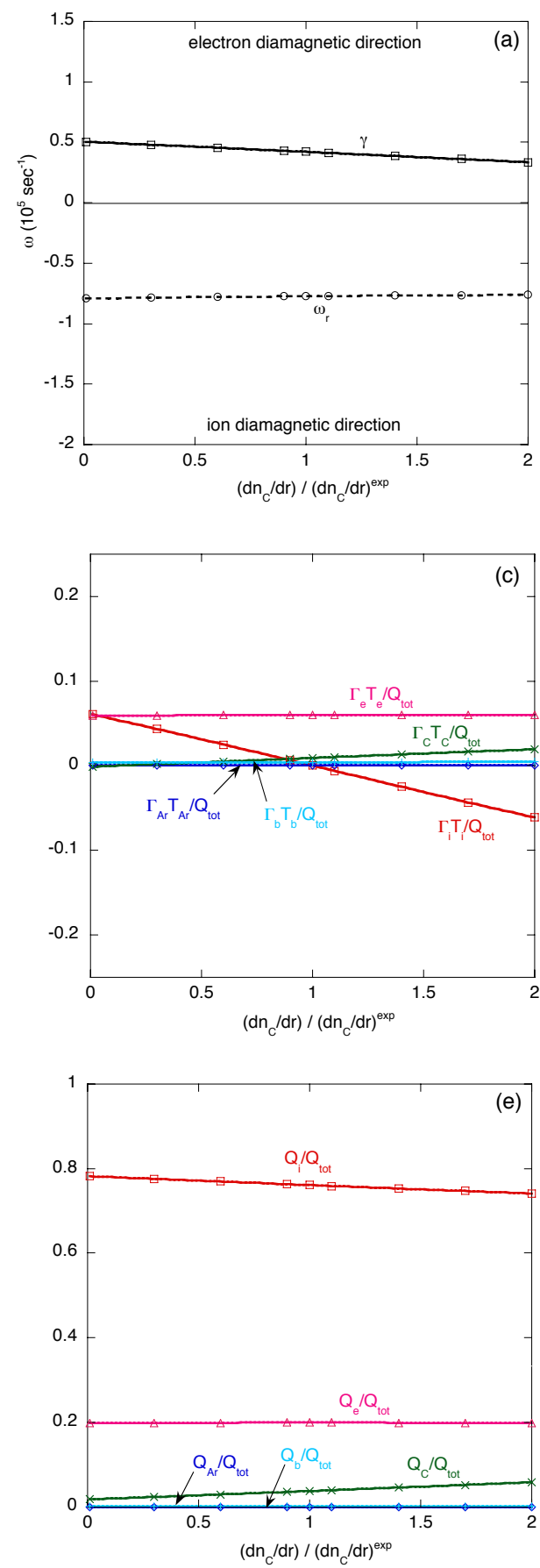

NSTX
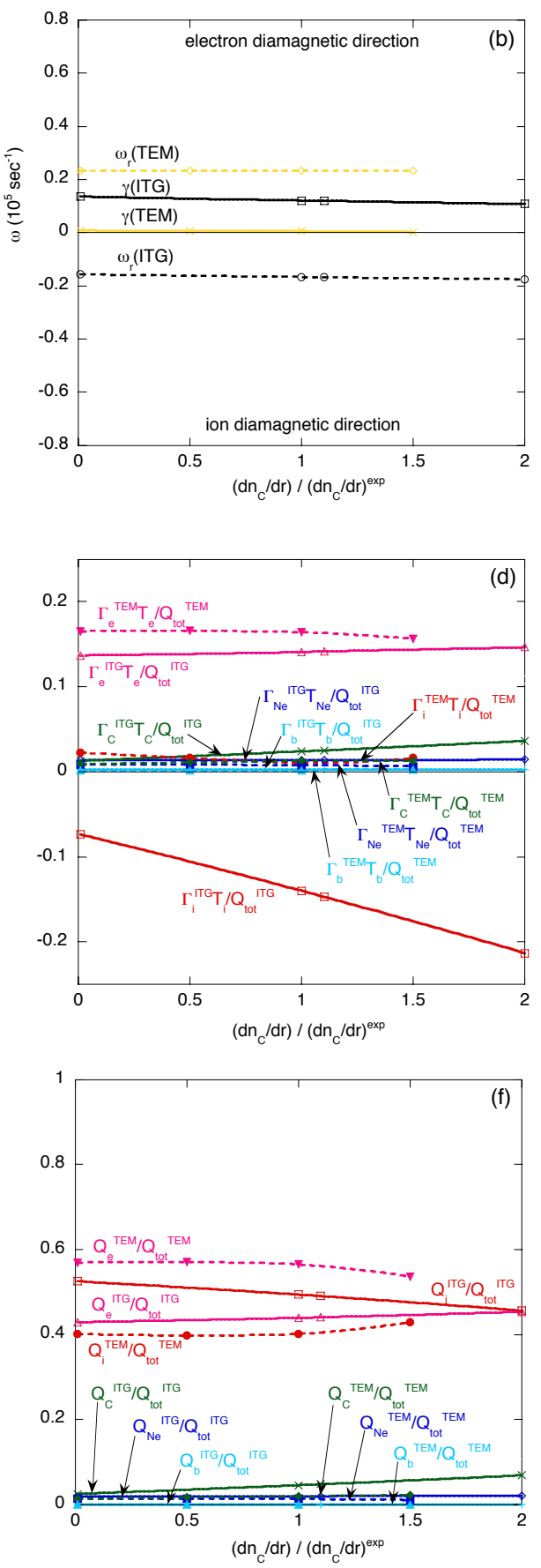

Fig. 4 
JET
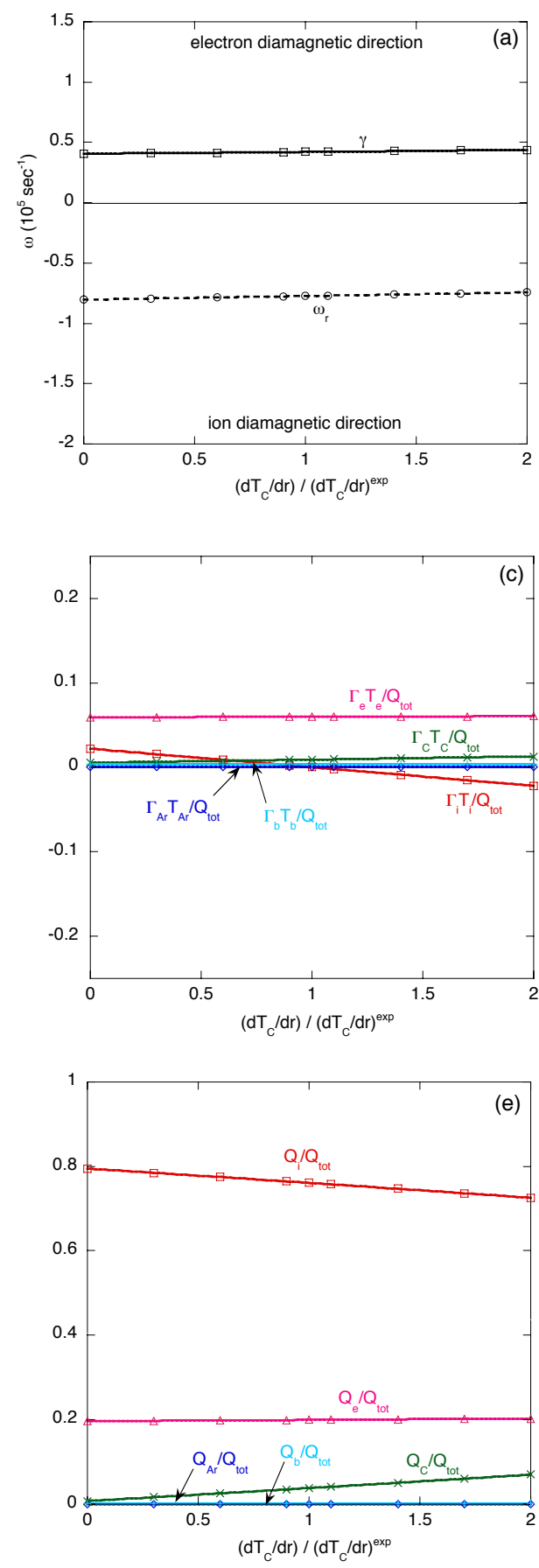

NSTX
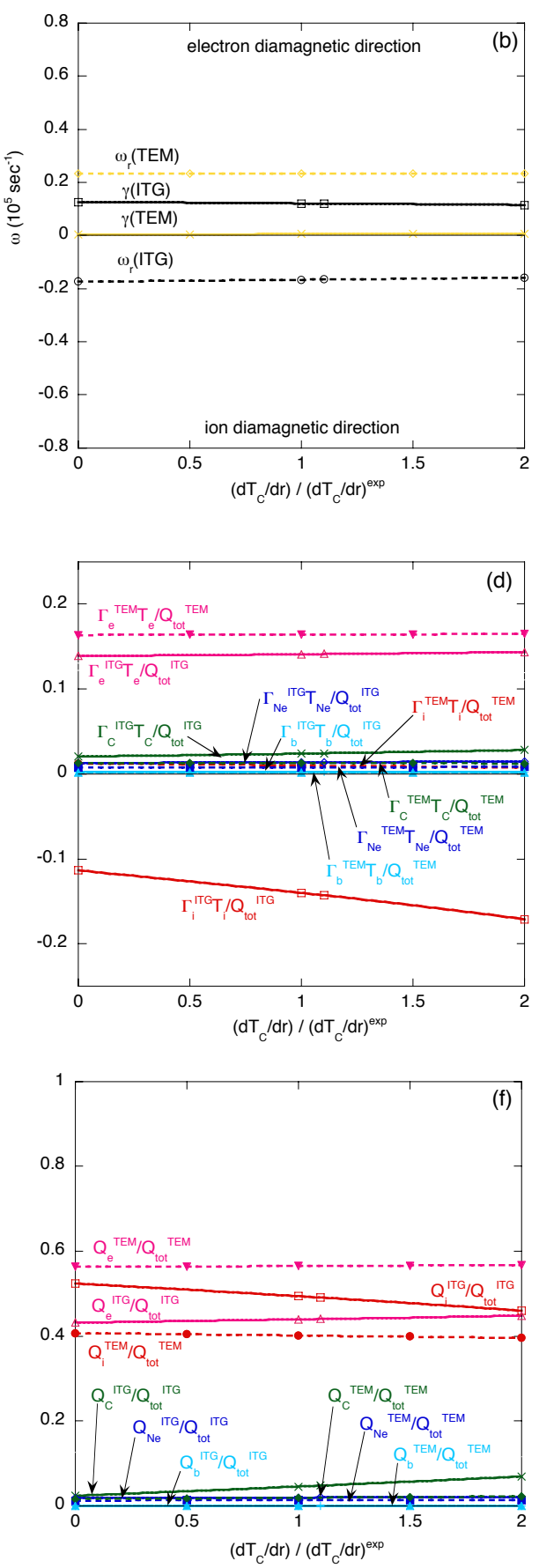

Fig. 5 
JET
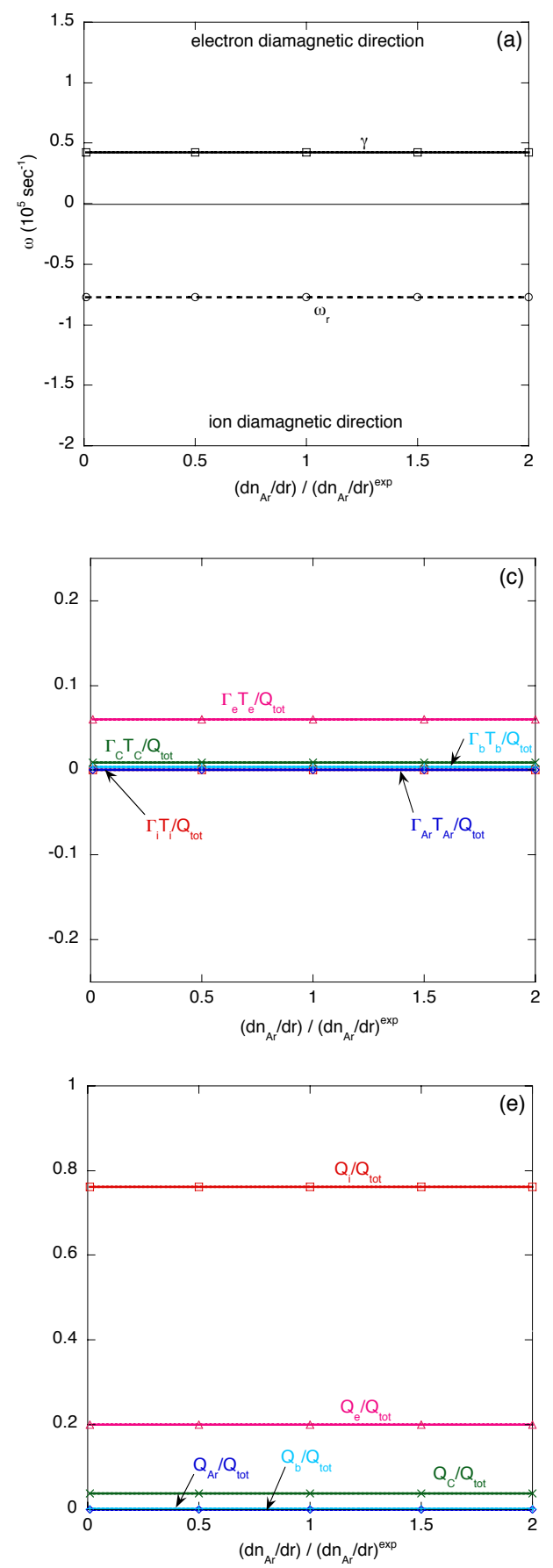

NSTX
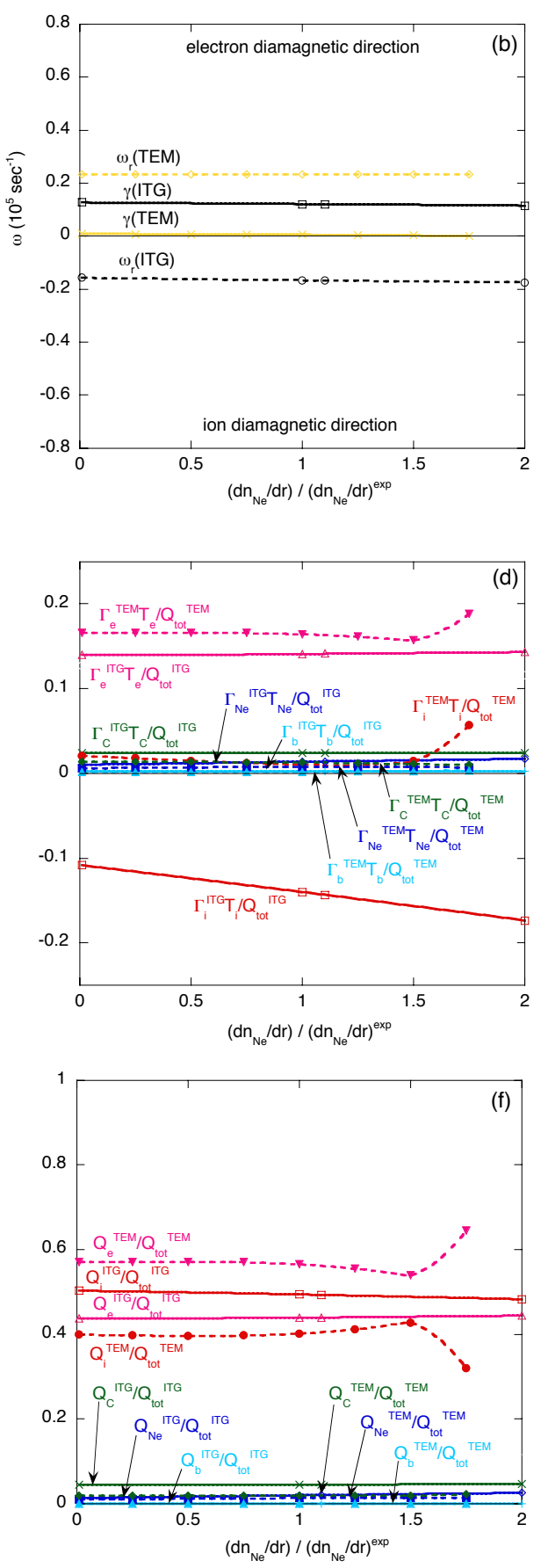

Fig. 6 
JET
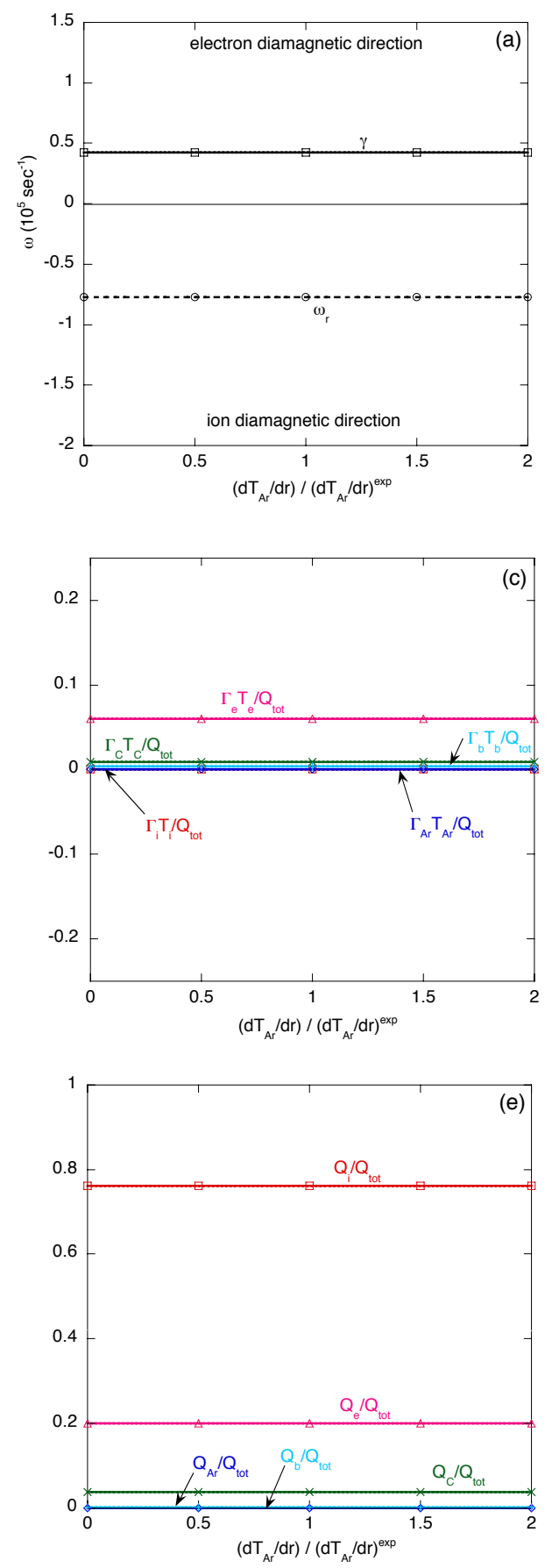

NSTX
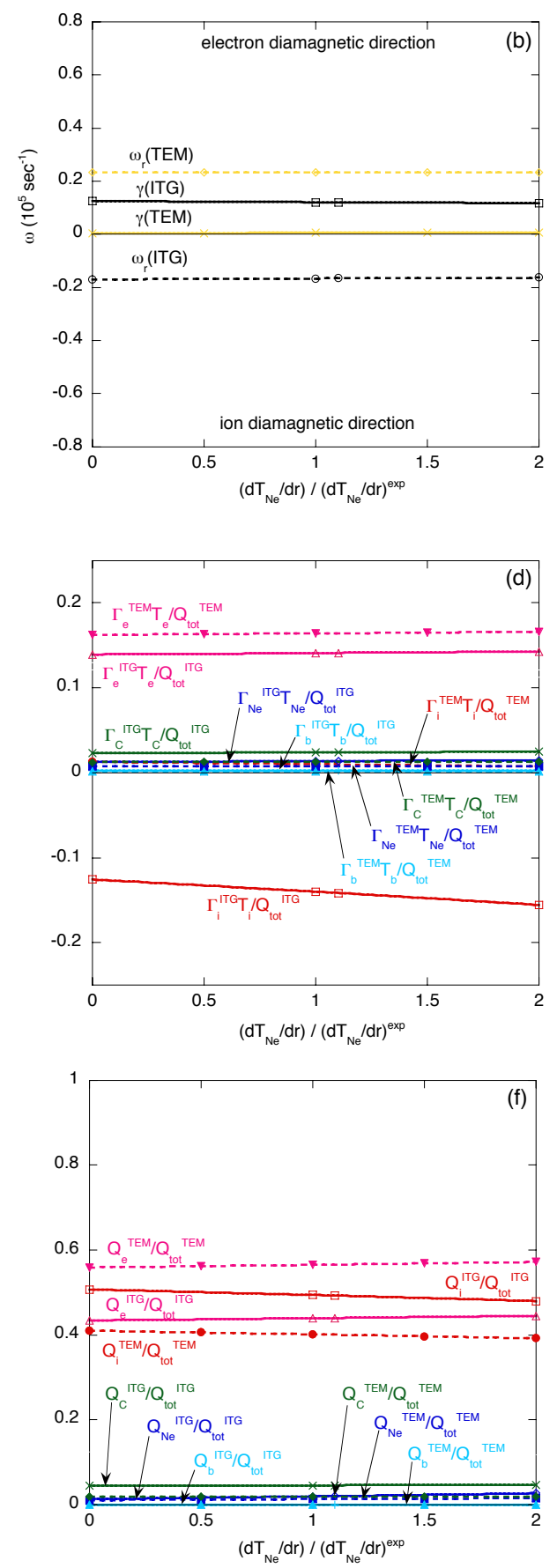

Fig. 7 
JET
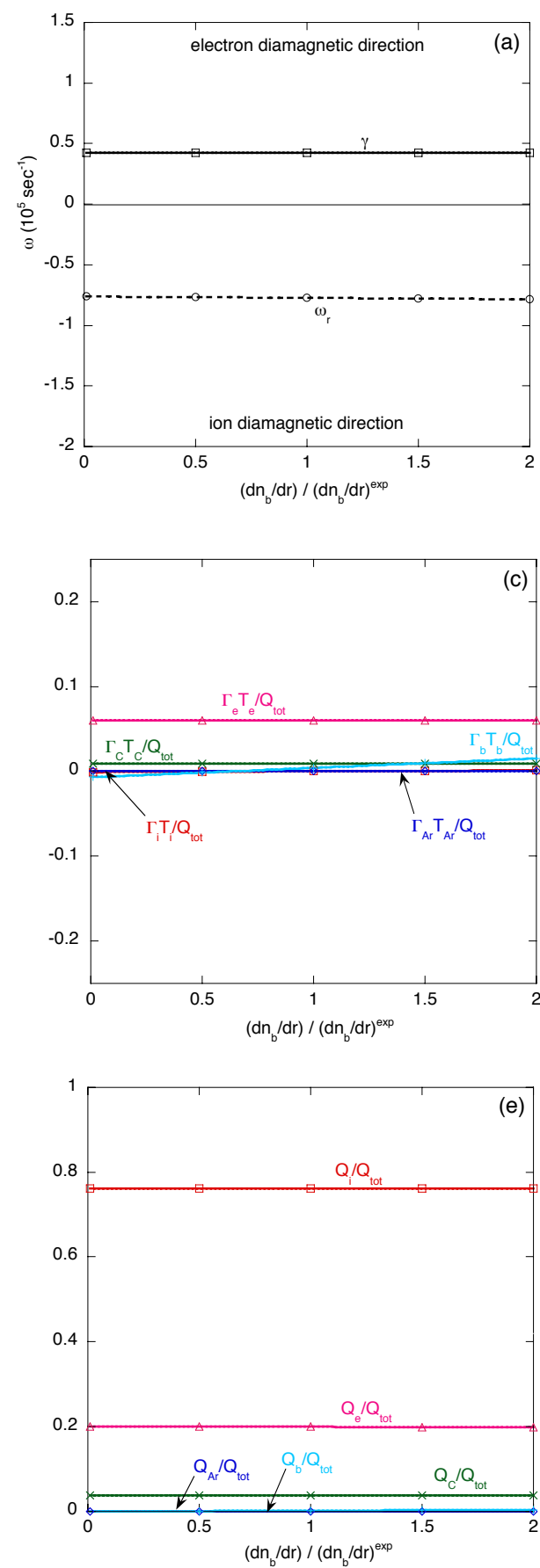

NSTX
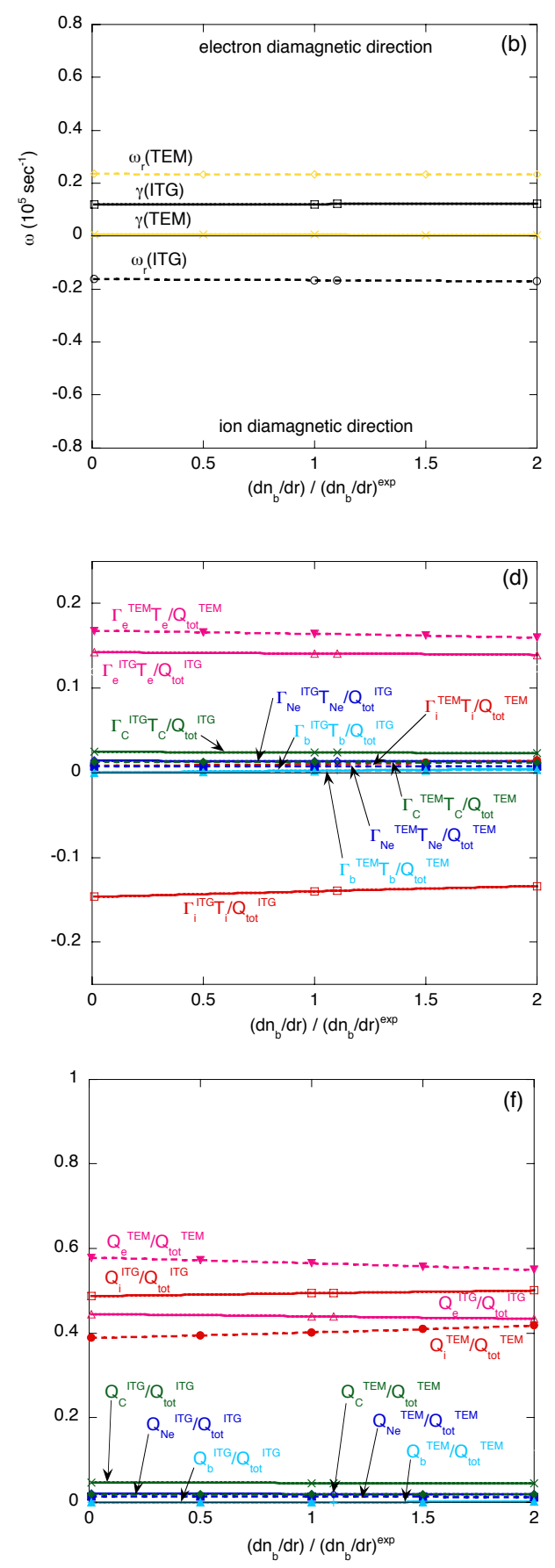

Fig. 8 
JET
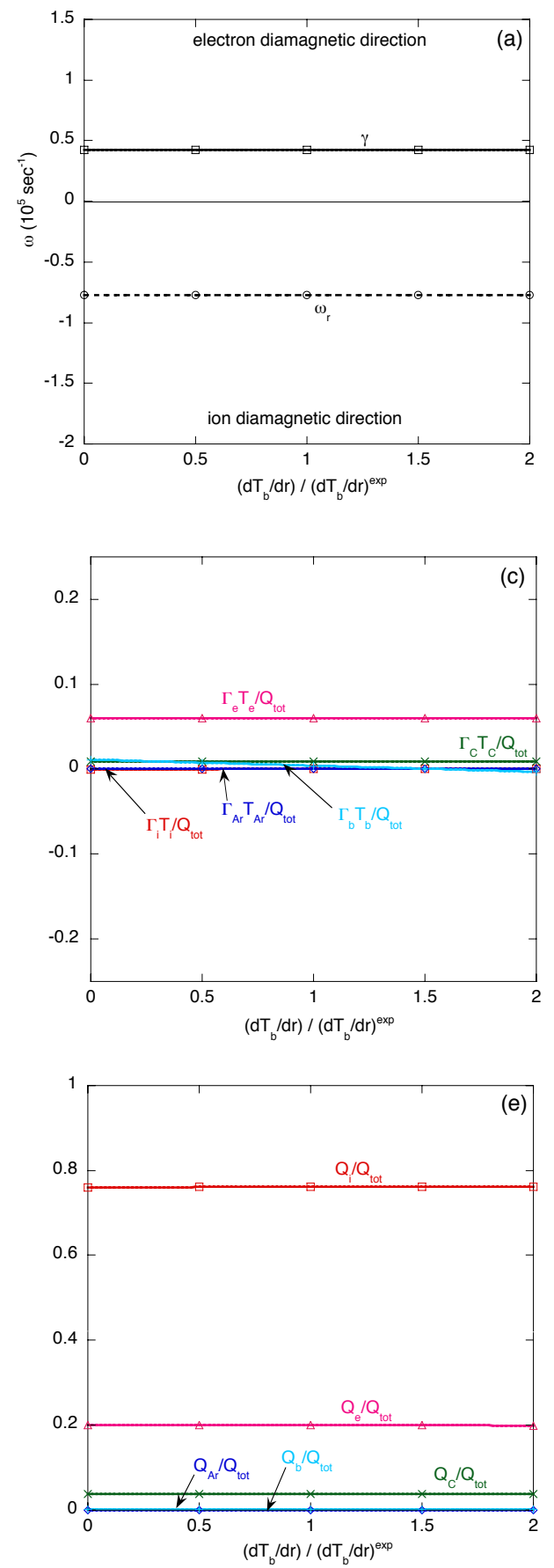

NSTX
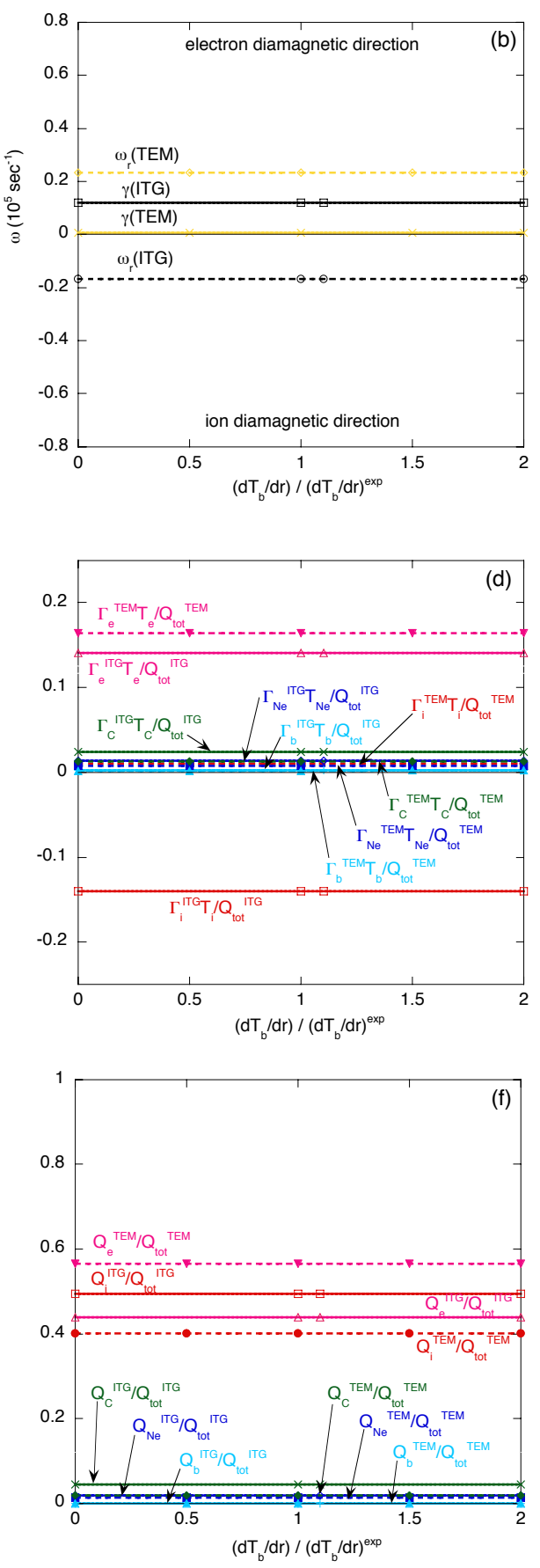


\section{External Distribution}

Plasma Research Laboratory, Australian National University, Australia

Professor I.R. Jones, Flinders University, Australia

Professor João Canalle, Instituto de Fisica DEQ/IF - UERJ, Brazil

Mr. Gerson O. Ludwig, Instituto Nacional de Pesquisas, Brazil

Dr. P.H. Sakanaka, Instituto Fisica, Brazil

The Librarian, Culham Laboratory, England

Mrs. S.A. Hutchinson, JET Library, England

Professor M.N. Bussac, Ecole Polytechnique, France

Librarian, Max-Planck-Institut für Plasmaphysik, Germany

Jolan Moldvai, Reports Library, Hungarian Academy of Sciences, Central Research Institute for Physics, Hungary

Dr. P. Kaw, Institute for Plasma Research, India

Ms. P.J. Pathak, Librarian, Institute for Plasma Research, India

Ms. Clelia De Palo, Associazione EURATOM-ENEA, Italy

Dr. G. Grosso, Instituto di Fisica del Plasma, Italy

Librarian, Naka Fusion Research Establishment, JAERI, Japan

Library, Laboratory for Complex Energy Processes, Institute for Advanced Study, Kyoto University, Japan

Research Information Center, National Institute for Fusion Science, Japan

Dr. O. Mitarai, Kyushu Tokai University, Japan

Dr. Jiangang Li, Institute of Plasma Physics, Chinese Academy of Sciences, People's Republic of China

Professor Yuping Huo, School of Physical Science and Technology, People's Republic of China

Library, Academia Sinica, Institute of Plasma Physics, People's Republic of China

Librarian, Institute of Physics, Chinese Academy of Sciences, People's Republic of China

Dr. S. Mirnov, TRINITI, Troitsk, Russian Federation, Russia

Dr. V.S. Strelkov, Kurchatov Institute, Russian Federation, Russia

Professor Peter Lukac, Katedra Fyziky Plazmy MFF UK, Mlynska dolina F-2, Komenskeho Univerzita, SK-842 15 Bratislava, Slovakia

Dr. G.S. Lee, Korea Basic Science Institute, South Korea

Institute for Plasma Research, University of Maryland, USA

Librarian, Fusion Energy Division, Oak Ridge National Laboratory, USA

Librarian, Institute of Fusion Studies, University of Texas, USA

Librarian, Magnetic Fusion Program, Lawrence Livermore National Laboratory, USA

Library, General Atomics, USA

Plasma Physics Group, Fusion Energy Research Program, University of California at San Diego, USA

Plasma Physics Library, Columbia University, USA

Alkesh Punjabi, Center for Fusion Research and Training, Hampton University, USA

Dr. W.M. Stacey, Fusion Research Center, Georgia Institute of Technology, USA

Dr. John Willis, U.S. Department of Energy, Office of Fusion Energy Sciences, USA

Mr. Paul H. Wright, Indianapolis, Indiana, USA 
The Princeton Plasma Physics Laboratory is operated by Princeton University under contract with the U.S. Department of Energy.

\author{
Information Services \\ Princeton Plasma Physics Laboratory \\ P.O. Box 451 \\ Princeton, NJ 08543
}

Phone: 609-243-2750

Fax: 609-243-2751

e-mail: pppl_info@pppl.gov

Internet Address: http://www.pppl.gov 